\title{
Regarding the Criteria for Planethood and Proposed Planetary Classification Schemes
}

\author{
S. Alan Stern and Harold F. Levison \\ Department of Space Studies, Southwest Research Institute, 1050 \\ Walnut Street, No. 426, Boulder, CO 80302, USA
}

\begin{abstract}
We examine the question of planetary classification, making recommendations both for the criteria by which planethood should be evaluated, as well as for more detailed physical and dynamical subtype classification schemes.
\end{abstract}

\section{Preamble}

The 1995 film entitled "The Englishman Who Went Up a Hill But Came Down a Mountain" is set in a small South Wales village during the first world war. The inhabitants of this village were very proud of their local mountain. Then two English cartographers appeared and decided that the mountain was no mountain at all, but only a hill. Indeed, the object in question was slightly too short to be a mountain. The villagers' solution involved moving a lot of dirt.

The main purpose of classification schemes is to allow the organization of thoughts. This is particularly true of reductionist classification schemes. Classification schemes have played and continue to play a useful role in astronomy because they allow us to put bodies into a structure with recognizable correlations of physical parameters.

There has been much recent discussion inside the planetary community, and in the public at large, over the status of Pluto. Despite the fact that many members of our community believed that such a debate was a matter of semantics, at times this discussion became very heated. Indeed, occasionally, the debate looked more like one between religious fanatics than scientists. While the discussion that has gone on may at times have centered on semantics, we believe that classification schemes play an important role in the scientific process.

One reason for developing a new classification scheme is to provide a framework for the evaluation of new ideas. We in planetary science have reached just such a time when new facts and new understandings motivate classification schemes for the bodies we study.

\section{Introduction}

What's in a name? That which we call a rose by any other name would smell as sweet. $-W m$. Shakespeare

The subject of how Solar System bodies should be classified seemed easy only two decades ago. Among the small bodies, comets showed tails, and asteroids did not. Regarding the planets, there were nine: 4 terrestrials, 4 gas giants, and Pluto. The story was pretty straightforward. 
But new facts intruded. Jupiter's and Saturn's interior structure and composition were found to be sufficiently different from Uranus' and Neptune's that aficionados subtyped them to "gas giants" and "ice giants." Pluto was found to be orbiting with a cohort of $>10^{5}$ miniature worlds in the Kuiper belt. Various planetary satellites, most small, but at least one substantial (i.e., Triton), were apparently captured from heliocentric orbit. Some bodies now classified as asteroids, like (2060) Chiron and (4015) Wilson-Harrington, have exhibited sporadic coma. An asteroid-like body (1996PW) was found coming from the Oort Cloud, the supposed long period comet reservoir. Pulsar planets were found, and so were "hot Jupiters" only a few solar radii from their stars; models showed giant planets can escape from their parent stars through mutual gravitational interactions. And so on, and so on...

These and other new facts blurred the simple boundaries and colloquial definitions that planetary astronomers had used in textbooks and in their research. In pretty good analogy to life, as our field grew more mature, simple black and white notions faded to a spectrum involving myriad shades of grey.

In this short paper we extemporize on our view of planetary classification. Regarding the definition of a planet, we will first set out to describe some attributes that we think any good classification scheme should offer. We will then discuss and dispose of several potential classification schemes that we believe are clearly flawed. Following this, we will discuss classification schemes that we think are good. And following that, we will add resolution to the suggested terminology, by speaking to the subject of both physical and dynamical sub-types.

\section{The Dilemma}

\section{If Jesus were Jewish, how come he's got a Mexican name? -Tom Waits}

We find it necessary to reject many of the proposed criteria for planethood. Why? As Table 1 shows, each such criteria fail in some obvious respect.

Table 1. Some Unsatisfactory Planetary Classification Criteria

\begin{tabular}{ll}
\hline Criterion & Problem/Counterexample \\
\hline $\begin{array}{l}\text { Presence of Satellites } \\
\text { Presence of an Atmosphere }\end{array}$ & $\begin{array}{l}\text { Omits Mercury, Venus; Can be time-dependent } \\
\text { Nominally Omits Mercury; } \\
\text { Problematic in what a minimum atmosphere is } \\
\text { Presence of a Magnetic Field } \\
\text { Omits Venus, perhaps Pluto } \\
\text { Allows all Solar System Objects, }\end{array}$ \\
Follows a Near-Circular Orbit & $\begin{array}{l}\text { Including comets, asteroids meteors, spacecraft, etc. } \\
\text { Omits Mercury, Pluto; } \\
\text { Problematic in what a minimum eccentricity is }\end{array}$ \\
Orbits a Star & $\begin{array}{l}\text { Omits ejected planets; allows dust; time-dependent } \\
\text { Omits Jupiter, Saturn, \& Neptune now, }\end{array}$ \\
Reflects More Light & $\begin{array}{l}\text { Hot planets after accretion, } \\
\text { cold Jupiter in the far future }\end{array}$ \\
\hline
\end{tabular}

Indeed, we find that no single criterion can adequately define the conditions for planethood. Instead, we argue, a series of criteria must be used in concert to form a good test. 


\section{The Goal}

Names are valuable; they can be your first source of insight into a character. -Spike Lee

Our goal is thus to achieve a classification algorithm, a sieve, that allows one to test any given body and determine if it is or is not a planet. ${ }^{1}$

The attributes that we wish such an algorithm to possess are that it should:

- Be physically based. That is, we want to achieve an algorithm that is based on physical tests. Therefore, it is unlikely that we will create a classification system that will allow for exactly 9 planets in the Solar System.

- Be determinable based on easily observed characteristics. That is, we want to achieve an algorithm that makes it easy to categorize all bodies, and which does not depend on poorly understood or poorly determinable concepts, such as mode of origin.

- Be quantitative. That is, the algorithm should produce results that are based on quantitative (read: numerically-based) properties or parameters of the bodies which it tests.

- Uniquely classify any given body. That is, no body should exit this algorithm with multiple classifications.

- Be deterministic. In particular, one does not want a body to change its status as a planet as a function of time, such as only when it possesses an atmosphere or magnetic field or satellites.

- Be robust to new discoveries. That is, the algorithm should be general enough to leave room for at least some unexpected discoveries (e.g., binary and trinary planets, bodies that have escaped their parent star, etc.).

- Be comprised of the fewest possible criteria. That is, the algorithm should be "fat free."

Additionally, while we do not consider it a requisite attribute of a viable test for planethood, we do think it desirable that any given test be reasonably backwards compatible. That is, it should attempt to avoid numerous reclassifications that would create confusion to lay people.

\section{Our Recommended Algorithm to Test Objects for "Planethood"}

There is a new margarine on the market called RUMOR, we named it this because it spreads so easily and quickly. -Anon.

In order not to violate any of the algorithm attributes described above, the individual criteria required to test for planethood must be precise, making sure they neither include red herrings, nor exclude obvious bodies in our Solar System. Further, it is our belief that planethood should be a genetic attribute of object class, rather than location, present circumstance, state, or exterior attributes.

Of course, one could simply define a planet as any body orbiting a star with a radius larger than some arbitrary value, say $1000 \mathrm{~km}$. This would be easy to

\footnotetext{
${ }^{1}$ There is of course a minimum degree of knowledge one must have about any given body to satisfy the requisites of such an algorithm.
} 
do, and it would satisfy most of the algorithm attributes given above. Perhaps this is what the IAU or other communities will ultimately do. However, we would find such a solution unappealing because it provides no insight into the nature of what it means for a body to be a planet, and it provides no physically meaningful insight into the nature of what planetary bodies are at their essence.

Our recommended algorithm for determining planethood, in contrast, attempts to provide just such insight. It is based on two simple criteria based on the body's mass; these criteria together trap planetary bodies between larger bodies called proto-stars, stars, or stellar remnants, depending on their stage of evolution, and smaller solid bodies we call planetesimals, rocks, and dust. Our formal definition is as follows:

A planetary body is defined as any body in space that satisfies the following testable upper and lower bound criteria on its mass: ${ }^{2}$ If isolated from external perturbations (e.g., dynamical and thermal), the body must:

1. Be low enough in mass that at no time (past or present) can it generate energy in its interior due to any self-sustaining nuclear fusion chain reaction (else it would be a brown dwarf or a star). And also,

2. Be large enough that its shape becomes determined primarily by gravity rather than mechanical strength or other factors (e.g., surface tension, rotation rate) in less than a Hubble time,${ }^{3}$ so that the body would on this timescale or shorter reach a state of hydrostatic equilibrium in its interior.

We have attempted to state these two criteria quite precisely, with certain caveats that ensure their generality. For example, we have used the word body to exclude distributed/ensemble astrophysical objects, such as molecular clouds, galaxies, etc., that are patently not planets. Further, our upper size limit says that a body that is large enough to (at any time) have generated low-temperature (e.g., deuterium) nuclear fusion, but has not had time to do so would in our definition not be considered a planetary body; this ensures that neither protostars nor stellar remnants (even if low in mass) ever qualify as planetary bodies. ${ }^{4}$

Similarly, our lower limit is based on the philosophy that the hallmark of planethood is the collective behavior of the body's mass to overpower mechanical strength and flow into an equilibrium ellipsoid whose shape is dominated by its own gravity. Notice also that this lower size limit does not insist on a shape that is set only by self-gravity, but rather one that becomes dominated by the body's gravity in a meaningful timescale; this allows rotation, mechanical strength, etc., to play minor roles, and it also allows for a period early in a planetary body's life when gravity may not yet have fully manifested itself to be the dominant force. This minimum mass criterion is best determined through observations. Our emphasis on the rule of gravity has the ancillary benefit of eliminating nearly

\footnotetext{
${ }^{2}$ Which, we note, can be determined by radial velocity techniques or from mutual planetary perturbations or from satellite orbits, or be estimated from a radius and a guess at density.

${ }^{3}$ We had to pick some long timescale, and stellar parent lifetime seemed inappropriate because it can give different results for the same planetary mass when orbiting stars with differing lifetimes.
}

${ }^{4}$ Though it may be necessary for very low mass bodies to obtain a spectrum in order to show they are not low-mass stellar remnants. 
spherical objects controlled by surface tension, electromagnetic or electrostatic forces, etc.

It is possible to analytically estimate when a solid body is massive enough to flow into an equilibrium ellipsoid owing to self gravity; a simple, approximate theory for this (e.g., Cole 1984) indicates this condition obtains if a non-rotating body's central pressure exceeds $\approx 2 \times 10^{8}$ dyne $\mathrm{cm}^{-2}$, which can be easily translated into a radius given a bulk density. More accurate minimum size calculations would explicitly take into account the variation in yield strength as a function of composition (rather than using density as a proxy for yield strength, as we have); such a refinement could result in factor of two changes in the minimum size criterion as a function of composition.

Figure 1 depicts this approximate guide to a minimum size boundary for planethood for bodies as a function of their bulk density, and compares this constraint to some illustrative bodies in our Solar System. Notice that large $\mathrm{KBO}$ and a few of the largest asteroids meet the criteria: while they may be "minor" planetary bodies, they are nonetheless, planetary bodies at their essence. As in life, we prefer to be inclusive, rather than exclusive.

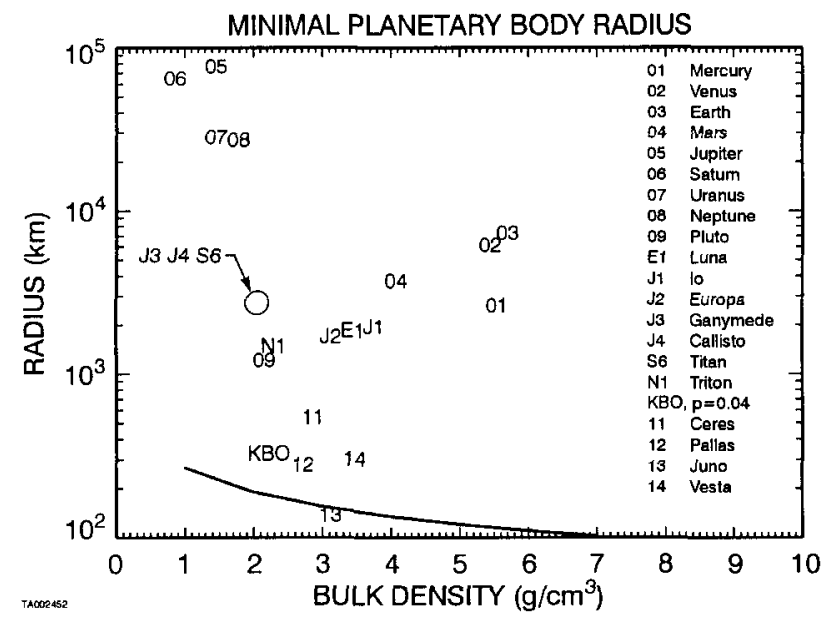

Figure 1. The minimum size boundary for a nonrotating planetary body, calculated from central pressure, as a function of bulk density is shown here, with numerous bodies in our Solar System for comparison.

This algorithm has some very nice advantages including: (i) planethood is based most fundamentally on a measurable or estimatable characteristic, mass; (ii) the algorithm can be quantitatively evaluated for any given body, giving a unique result (yes or no), which is not a function of time for the body; (iii) planethood does not depend on where a body is located; and (iv) planethood is insensitive to issues such as whether a body has satellites, a magnetic field, or an atmosphere.

By our criteria, planetary bodies span a wide range of masses ranging upward from $\approx 10^{-3} M_{\oplus}$ to almost $\approx 10^{4} M_{\oplus}$. While this is a larger mass range than stars $\left(\approx 6 \times 10^{-3} M_{\odot}\right.$ to almost $\left.\approx 10^{2} M_{\odot}\right)$, it is not unlike the mass range of galaxies $\left(\approx 10^{7} M_{\odot}\right.$ to almost $\left.\approx 10^{13} M_{\odot}\right)$. 
Notice that by our definition, both large satellites of planets, as well as bodies freely floating in interstellar space, qualify as planetary bodies (a term we consider to represent the intrinsic nature of a body). We therefore propose the following nomenclature to differentiate between the dynamical circumstances in which a planetary body is found: cases: A planet is any planetary body on a bound orbit around a single or multiple star system; by our definition, the classical 9 planets, as well as a few of the largest asteroids, and very large KBOs, qualify. A planetary-scale satellite is any planetary body orbiting a larger planetary body on a bound orbit; examples of planetary-scale satellites include Luna, the Galilean satellites, Titan, and Triton. ${ }^{5}$ An unbound planet is any planetary body not bound to a single or multiple star system. Obviously, planetary bodies can, through dynamical evolution, change status among these cases; for example, because Triton appears captured into orbit about Neptune, this planetary-scale satellite was formerly a planet in its own right.

\section{Toward Higher Resolution: Proposed Dynamical Classification}

It is a matter dominated universe- Those that matter, dominate. -Yvonne Pendleton

Beyond the strict inclusion/exclusion of bodies in the planetary class, it is useful to achieve a descriptive level of further classification that denotes whether or not a specific body is in some sense dynamically important to the system in which it resides. Indeed, the planetary bodies in heliocentric orbit include the terrestrial planets, ice giants, giant planets, large asteroids, and (if KBO albedos are indeed near $4 \%$ as we assumed) large KBOs like Pluto, $1996 \mathrm{TO}_{66}$, and $2000 \mathrm{~EB}_{173}$. And analogous situations apply within satellite systems of the giant planets. Because such smaller bodies clearly play a dynamically different role in the Solar System than the large bodies that architecturally shape the system, distinguishing between the various bodies on some dynamical basis is both useful and desirable.

The largest planetary bodies dynamically control the region surrounding them. Nearby small bodies are on unstable, transient orbits, or are locked in mean motion resonances or in satellite orbits. ${ }^{6}$ We believe such a distinction is likely to be a common one in extra-solar planetary systems as well.

Our goal for a dynamical classification scheme is thus to determine whether any given body is dynamically important to the system in which it is found. Hence, we define an überplanet as a planetary body in orbit about a star that is dynamically important enough to have cleared its neighboring planetesimals in a Hubble time. And we define an unterplanet as one that has not been able to do so. It is important to note that, unlike the physical criteria for planethood, these purely dynamical criteria cannot be an intrinsic aspect of a body, but is necessarily dependent on the environment and dynamical context in which a body finds itself.

\footnotetext{
${ }^{5} \mathrm{~A}$ double planet would be a system consisting of a planet and a satellite which is massive enough to place their mutual barycenter outside of the primary, and between the two bodies. Pluto-Charon is the only example in our Solar System.

${ }^{6}$ For example, the near-Earth objects are transient bodies with median lifetimes of only 10 million years, while Trojans and Plutinos are locked into mean motion resonances with Jupiter and Neptune, respectively.
} 
It is crucial for the reader to note that, owing to the coupled nature of $\mathrm{N}$-body dynamical systems, it is not possible to write down a simple formula for whether a planetary body will clear its neighboring region. The only truly viable method for determining whether a planetary body is über or unter is to perform a series of numerical integrations.

Nevertheless, to provide a simple, heuristic feel for the nature of our proposed dynamical classification scheme, it is possible to construct a crude estimate of whether a planetary body can dynamically clear its local environment. To do so, we apply the techniques developed by Öpik (1951), which estimate the probability $P$ per orbit that a small body with semi-major axis $a$ will pass within distance $b$ of a planet (assumed to be on a circular orbit) is:

$$
P=\frac{s^{2} U}{\pi \sin i\left|U_{x}\right|}
$$

where

$$
s^{2}=\left(\frac{b}{a}\right)^{2}\left(1+2 G M_{p} / b V_{\infty}^{2}\right)
$$

and $\theta$ is the deflection angle of the small body during an encounter, so that:

$$
\tan \left(\frac{\theta}{2}\right)=\frac{\mu a}{b U^{2}} \equiv \Gamma \text {. }
$$

Here $\mu=M_{P} / M_{\odot}, G M_{p}$ is the gravitational mass of the planet, $V_{\infty}$ is the relative velocity of the small body and planet, $U=V_{\infty} / V_{p}$ (where $V_{p}$ is the planet's orbital velocity), $i$ is the relative inclination of the orbital planes of the small body and planet, $U_{x}$ is the radial component of $U$, and $b$ is the impact parameter of the encounter.

We can now estimate the likelihood $\Lambda$ that in a Hubble time $(\tau)$ a small body will suffer an encounter with a planet that leads to a deflection of a particular magnitude, $\Gamma$. This is:

$$
\Lambda=\frac{\mu^{2}}{a_{p}^{3 / 2}}\left[\frac{\tau \sqrt{G M_{\odot}}(1+2 \Gamma)}{2 U^{3} \pi^{2} \sin (i)\left|U_{x}\right| \Gamma^{2}}\right] .
$$

Notice that the term within the brackets is only a function of $\Gamma$ and the orbital elements for the small body, and is independent of the physical characteristics of the planet. The boundary between überplanets and unterplanets occurs at $\Lambda=1$. One evaluates $\Gamma$ where $\theta \sim 1$ radian; notice that, because $\Gamma$ is always of order unity, $(1+2 \Gamma) / \Gamma^{2}$ is itself of order unity.

It is not possible to evaluate these equations without making assumptions about the orbits of the small bodies in the system. The term between the square brackets is a function of the small body's eccentricity, inclination, and the ratio of its semi-major axis to that of the planet. Fortunately, we have a sample of small bodies at our disposal that we can use as a test case, the asteroid belt. By taking the orbits of the known main belt asteroids, $a_{p}$ equal to the semi-major axis of Ceres, $\Gamma=1 / 2(\theta \sim 1$ radian $)$, and $\tau=12 \mathrm{Gyr}$, the average value of the term between the square brackets is $1.7 \times 10^{16}$ years. Applying this in Eqn. (4), we find that $\Lambda=4 \times 10^{-3}$ and $8 \times 10^{-4}$ for Pluto and Ceres, respectively, while $900<\Lambda<10^{9}$ for the more massive members of our planetary system. 
A comparison between different bodies in our Solar System can be made by studying the term $\mu^{2} / a_{p}^{3 / 2}$. Such a comparison can be found in Figure 2. From a dynamical standpoint, our Solar System clearly contains 8 überplanets and a far larger number of unterplanets, the largest of which are Pluto and Ceres. Interestingly, were Luna in heliocentric orbit at $1 \mathrm{AU}$, dynamically capable of clearing its zone; as a result it would most easily be classified as an überplanet.

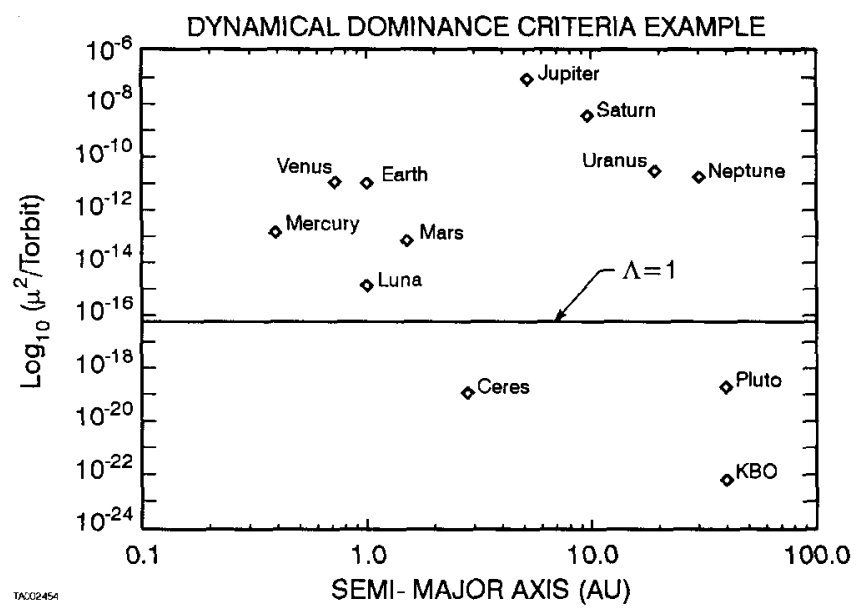

Figure 2. The dynamical dominance criteria $\mu^{2} / T_{\text {orbit }}$ is plotted for a variety of planetary bodies in our Solar System; the solid horizontal line here delineates $\Lambda=1$ (the über vs. unter boundary). For reference, if $\Lambda=1$, then $\mu^{2} / T_{\text {orbit }}=6 \times 10^{-17}$, so that at $1 \mathrm{AU}$ (5 AU), $\mu=$ $7.7 \times 10^{-9} M_{\odot}\left(\mu=3.8 \times 10^{-8} M_{\odot}\right)$.

\section{Toward Even Higher Resolution: Proposed Physical Classification}

A man that should call everything by its right name, would hardly pass the streets without being knocked down. - Lord Halifax

The proposed criteria for admitting (or rejecting) any body into (or from) the class of planetary bodies has many attractive features, most notably the shared physical attribute that the bodies which meet the criteria are shapedominated by gravity, but not so large as to ever have or in the future generate energy via a fusion chain reaction.

However, owing to the large mass range which satisfies our criteria, we believe it is also useful to define physical types which allow astronomers to categorize planetary bodies in various planetary systems. Such finer resolution into types of course requires knowing more things about the bodies.

Our proposal in this regard is a two-parameter (size, composition) matrix of subtypes that loosely parallels astrophysical nomenclature for stars. Regarding size we propose the subtypes, Subdwarf, Dwarf, Subgiant, Giant, and Supergiant, spanning the entire range from a minimum to a maximum sized planetary body. Regarding composition, we propose Rock, Ice, and Hydrogen gas, based on the primary constituent in the planetary body; compositional types list could if necessary be expanded, e.g., owing to new discoveries. Table 2 shows how the 
known planetary bodies in our Solar System and around other stars map into these types. KBOs = Kuiper Belt Objects. Planetary scale satellites have been left off this table for clarity only ${ }^{7}$; they can easily be included. But as one example, Titan, a planetary body by virtue of its mass, would be considered an icy subdwarf satellite in this scheme.

Table 2. A Roster of Known Planets by Type (ca. 2000)

\begin{tabular}{llllll}
\hline Comp. & Subdwarf & Dwarf & Subgiant & Giant & Supergiant \\
$\mathrm{M}<$ & $0.03 \mathrm{M}_{\oplus}$ & $10^{\mathbf{1}} \mathrm{M}_{\oplus}$ & $10^{2} \mathrm{M}_{\oplus}$ & $10^{\mathbf{3} \mathrm{M}_{\oplus}}$ & $10^{\mathbf{4 . 5} \mathrm{M}_{\oplus}}$ \\
\hline Rock & Largest Asteroids & Mercury, Mars & & & \\
& Numerous KBOs & Venus, Earth & & & \\
& Pluto, Charon & PSR1257+12C & & & \\
& PSR1257+12A,B & & & & \\
Ice & Numerous KBOs & & Uranus, & \\
& & & Neptune & & \\
Hydrogen & & & Saturn & Jupiter & \\
\hline
\end{tabular}

We believe that this secondary descriptor, i.e., the physical attributes, should follow the dynamical attributes in importance in any planetary or satellite system. Together, the combination of dynamical and physical descriptors allows for a rich and flexible nomenclature, allowing for everything from übergiants like Jupiter to unter-subdwarfs like Pluto to über-dwarfs like Earth to (hypothetical) unter-giants in Oort Clouds.

\section{Coda}

Just because your doctor has a name for your condition, doesn't mean he knows what it is. -Franz Kafka

Planetary scientists and astronomers are rapidly gaining an appreciation of the fact that old notions of what is and is not a planet are (for understandable historic reasons) both naïve and restrictive. We have attempted to contribute some (hopefully useful) new thinking to the issue of planetary nomenclature.

Acknowledgments. We thank Brian Marsden for motivating us to write this paper. Numerous colleagues, including Mike A'Hearn, Bill Bottke, Luke Dones, Martin Duncan, Dan Durda, Steve Howell, Joel Parker, and Bill Ward contributed valuable comments on this paper. We also thank Don Davis, Allessandro Morbidelli, and Hans Rickman, as each contributed helpful comments as reviewers.

\section{References}

Cole, G.H.A. 1984, The Physics of Planetary Interiors, Adam Hilger Ltd., Bristol, $204 \mathrm{pp}$.

Öpik, E.J. 1951, Proc. R. Irish Acad., 54A, 165

\footnotetext{
${ }^{7}$ We exclude planetary bodies that are satellites from this table in order to save space; the planetary bodies not shown include the following satellites in orbit around planets: Galileans, Titan, Triton, and Luna.(with mass boundaries and major composition specified for each)
} 